\title{
METHODOLOGY OF NEUROPSYCHOLOGICAL ASSESSMENT: QUALITATIVE (METASYNDROMAL ANALYSIS OF COGNITIVE DEFICIT STRUCTURE) AND QUANTITATIVE (PSYCHOMETRIC ESTIMATE) ASPECTS
}

Yuri V. Mikadze

Lomonosov Moscow State University Moscow

\begin{abstract}
Neuropsychological evaluation is a necessary step that precedes neurorehabilitation. It implies both qualitative and quantitative assessment of patient's possible cognitive deficit. Qualitative approach aims to define the structure of the cognitive deficit while quantitative procedures give estimate of the magnitude of the deficit. Complex pathology affects multiple brain regions and reveals in a series of neuropsychological syndromes associated with these brain areas. Metasyndrome analysis is proposed as an approach to understanding the complex cognitive impairments. A new concept of metasyndrome is introduced in order to describe these repetitive patterns of observed cognitive deficits. Quantitative estimate allows to determine the degree of cognitive disorder and the dynamics of its change.
\end{abstract}

Keywords: neuropsychology, metasyndromal analysis, neuropsychological evaluation

\section{Introduction}

Neuropsychological assessment provides guidance to further settings of neurorehabilitation based on individual observed cognitive profile. However there is a question whether the specificity of individual cognitive deficit might rely only on psychometric evaluation of separate cognitive functions. In order to serve the purpose of neurorehabilitation neuropsychological assessment should combine both qualitative and quantitative approaches. The first implies comparison of specific cognitive impairments while qualitative approach focuses on the magnitude of the observed deficits.

Syndrome analysis is an example of a qualitative approach to the description of the complex structure of cognitive deficit. It determines the procedure of the assessment and the following data interpretation 
binding observed symptoms with their possible brain origins. Different components of the neuropsychological diagnosis might be brought together by the concept of neuropsychological factor first introduced by A.R. Luria.

The concept of neuropsychological factor was used to map different symptoms to the localization of brain lesions that presumably caused these symptoms. Neuropsychological factor couples aspects of cognitive functioning with the brain anatomy creating a unit for further analysis.

Neuropsychological factors relate to:

- localization of different brain regions (anatomical aspect) and to the functions of these brain regions (neurophysiological aspect).

- different components of cognitive functions - particular psychological qualities produced by the activity of appropriate neuronal substrate - included in the general psychological structure of cognitive functions (psychological aspect).

Synchronous and sequential consolidation of brain areas in the ontogenesis leads to the development of various functional brain systems (Anokhin, 1980; Philimonov, 1974). Cognitive functions rely on the integrative work of different brain regions mediated by the inter and intraconnections between these functional systems.

Joint functioning of different brain regions (functional systems) emerges as a temporal-spatial structure that might be described in Luria's framework through the concept of neuropsychological factors. Factors refers to the principle components of high order cognitive functions.

Neuropsycological factors relate to neuropsychological syndromes genuine constellations of different cognitive deficits. The localization of the lesion determines the symptoms and they in turn illustrate the breakdown of a specific neuronal mechanism associated with that particular brain region. Behaviorally we might observe the combination of cognitive symptoms that all resulted from the same altered neural mechanism and that all have in common some psychological components.

According to K. Yaspers, clinical pictures of the disorders with the same origin, the same basic psychological structure, and associated with the same brain pathology, constitute organic nosological units. In A.R. Luria's methodology neuropsychological syndrome illustrates this kind of nosological unit as a result of the damage to a particular brain region. In the neurorehabilitation perspective neurophysiological syndrome 
might be used as a core unit in the description of qualitative and quantitative aspects of patient's cognitive deficit.

Qualitative evaluation of the cognitive deficit is provided through the factor analysis of observed neuropsychological syndromes. Outlined factors are then correlated with the corresponding brain regions and their functions.

Quantitative analysis includes further classification and ranging of the neuropsychological syndromes based on their prevalence and magnitude of their constituted symptoms.

Applying system theory framework to the neuropsychological syndromes, the cognitive deficit represents a pattern of impaired functioning of different components within the cognitive system that show up in the neuropsychological syndrome. Furthermore, the disruption of inter and intra-connections between different functional systems of the brain results in different constellations of neuropsychological syndromes.

In order to implement the presented approach to the description of cognitive deficits in case of diffuse complex brain pathology new methodological constructs should be developed. In every individual case neuropsychologist has to deal with neuropsychological syndromes associated with the primary impairments as well as with the compensatory changes in cognitive functioning that taking together form a stable pattern. Further classification of revealed syndromes based on their range and magnitude might be summarized in terms of "metasyndrome analysis".

\section{Materials and Methods}

One of the examples of the application of metasyndromal analysis was a neuropsychological assessment of children and adolescents with focal epilepsy (performed together with T.Y. Gogberashvili). Total of 82 patients, in the age of 6-16 were assessed: 34 patients had been diagnosed with focal frontal epilepsy, 28 - with focal temporal epilepsy and 20 patients had focal occipital and parieto-occipital epilepsy. According to EEG data 44 children had a well localized epileptic focus in one of the hemispheres, 38 children had a bilateral site of epileptic focus.

Neuropsychological assessment consisted of A.R. Luria test battery that included: clinical interview, tests on executive functions, memory, 
perception, language and speech and motor functions. Performance on each test was evaluated using a 3-point scale (from 0 - for no deficit to 3 - inability to perform the task). The observed symptoms were then ranged in their magnitude and clusters of syndromes were computed. The combinations of syndromes were further analyzed both qualitatively (factor composition) and quantitatively (the degree of intensity) applying proposed metasyndrome methodology.

\section{Results}

The results of the assessment could not be described in terms of local specific syndromes associated with a particular area in the brain. Instead, combinations of syndromes for different epileptic focuses were deliniated depending on their prevalent symptoms and their magnitutde.

Metasyndromes were defined based on the localization of the epileptic focus and the time of the first onset of the disorder (after the age of three) regardless the age of a child at the time of the evaluation. Each metasyndrome consisted of the primary syndrome directly related to the epileptic focus and several other less prominent syndromes that did not directly point out to the epileptic brain region. For each type of focal epilepsy epileptic focus had different impact on the other brain regions. Epileptic focus located in frontal, temporal, occipital or parieto-occipital region had primary impact on the regions functionally connected with the affected area.

For example, in case of the focal left temporal epilepsy metasyndrome included a range of syndromes associated with the dysfunction of different brain regions: prevalent symptoms signaled temporal lobe dysfunction (factors of verbal auditory perception and verbal memory span), second order symptoms were associated with temporo-parieto-occipatal area (spatial and quasi-spatial factors) and third order symptoms refered to prefrontal and motor areas disturbances (factor of programming and executive control, kinetic factor). The least prominent were the symptoms emanating from the subcortical structures and dyencephalon (factor of arousal-inactivation).

The histogram shows an example of a metasyndrome (a pattern of ranged syndromes) observed in the case of the focal left temporal epilepsy in 12-years old girl (Fig. 1). 


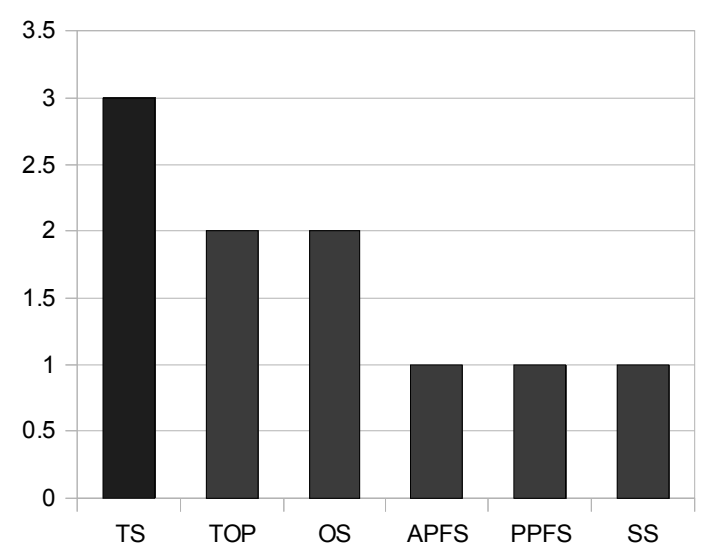

Figure 1. Metasyndrome Components

Different syndromes are presented on the $\mathrm{X}$-axis ranged from the most prevalent (purple color) to the least: temporal (TS), temporo-parietal-occipital (TOP), occipito-temporal (OS), anterior and posterior prefrontal (APFS and PPFS) and subcortical (SS) syndromes. On the Y-axis is plotted the magnitide of the deficit based on the test performance

In comparison with the temporal focal epilepsy frontal focal epilepsy revealed metasyndrome that has a frontal syndrome as a main component. The observed symptoms could be ranged from the most to the least prevailing: symptoms originating from anterior and posterior prefrontal areas (factor of executive control and programming, kinetic factor), temporal lobe symptoms (factors of auditory verbal perception and verbal memory span), occipito-temporal and temporo-parieto-occipital symptoms (spatial and quasi-spatial factors), and the symptoms from subcortical structures (factor of arousal and inactivation).

\section{Conclusions}

According to our data, in the example reviewed above, each form of the focal epilepsy (temporal, frontal, occipital and parieto-occipital) resulted in its own metasyndrome. The variations in syndromes within the metasyndrome was less than the variations between metasyndromes that might speak for the stable patterns of metasyndromes associated with a particular epileptc focus. We suppose that pathological neuronal 
activity spreading out through different pathways affects functioning of the brain structures involved in the epileptic circuit. This altered functioning shows up in the variety of cognitive symptoms. Although these symptoms cannot be related to a unit brain lesion they still form a stable pattern. The described patterns of distorted cognitive functioning associated with different brain regions in different epileptic focuses should be taken into consideration when developing neurorehabilitation treatment plans for children with focal epilepsy.

Neuropsychological assessment necessarily precedes neurorehabilitation. Luria's approach to the assessment of patients with brain lesions consists of, firstly, describing the deficits in high-order cognitive functions, secondly, determining the damaged brain areas responsible for these symptoms and finally defining a neuropsychological syndrome as a genuine constellation of these symptoms.

Two practical issues come up with the assessment. One is the need of defining the structure of the neuropsychological deficit that guides the development of the rehabilitation plan and evaluation of the progress.

Second is related to the localization of the lesion and more importantly to the detection of functionally affected brain regions that cannot be observed using structural imaging techniques.

Original syndrome analysis proposed by Luria was based on the description of syndromes that result from the well-defined local lesions. Diffuse brain lesions (e.g. TBI, MS) lead to multiple deficits that don't converge into a unit syndrome but could rather be described as a complex of syndromes associated with affected brain regions.

Global pathological conditions (stroke or epilepsy) secondary disturb the normal functioning of different brain regions functionally and morphologically connected to the prime lesion. These dysfunctions show up in the assessment in form of mild to severe syndromes. Combinations of these syndromes might reveal a stable pattern. A new concept of metasyndrome is introduced in order to describe these repetitive patterns that reflect both the effects of lesion and functionally impaired brain regions on cognition and behavior.

Metasyndromal approach to the analysis of the disorder structure in this case should be considered as further development of syndromal (factorial) analysis, developed by A.R. Luria and his successors.

Since psychometric tests are usually aimed at general quantitative estimate of a particular cognitive function and does not allow to differ- 
entiate the status of separate components of these cognitive functions, therefore neuropsychological assessment should necessarily combine general neuropsychological syndromal analysis (aimed at revealing the structure of the disorder) and psychometric methods (aimed at quantitative estimate of cognitive deficit). This allows to solve such problems in neuropsychological assessment as developing further rehabilitation procedures and the description of the degree of the disorder, the dynamics of their change in the course of treatment and rehabilitation.

\section{References}

Anochin, P.K. (1980). Uzlovye voprocy funktsional'nykh system [The central questions of functional systems]. Moscow: Nauka.

Luria, A.R. (1966). Higher Cortical Functions in Man. New York: Basic Books.

Philimonov, I.I. (1974). Izbranye trudy [Collected works]. Moscow: Meditsina.

Yaspers, K.J. (1997). Obshchaya psikhopatologiya [General psychopathology]. Moscow: Praktika. 